\title{
Fabrication of Heparinized Mesoporous Silica Nanoparticles as Multifunctional Drug Carriers
}

\author{
Yongxiang Zhang, Xinmin Liu, Yizhong Lu, Jincai Wang, Tingfang Dong, and Xiaohong Liu
}

The First Affiliated Hospital, Xinxiang Medical University, Xinxiang 453100, China

Correspondence should be addressed to Yongxiang Zhang; zhangyongxiang75@sina.com

Received 28 January 2013; Accepted 1 March 2013

Academic Editor: Yufang Zhu

Copyright ( 2013 Yongxiang Zhang et al. This is an open access article distributed under the Creative Commons Attribution License, which permits unrestricted use, distribution, and reproduction in any medium, provided the original work is properly cited.

In this study, heparinized multifunctional mesoporous silica nanoparticles were successfully synthesized and characterized. The new material not only maintains intrinsic functions of bare magnetic and fluorescent mesoporous silica materials such as targeting, imaging, and sustained release of drugs, but also generates several novel activities such as the enhancement of biocompatibility, selective loading drugs, and dual loading of anticancer drug and bFGF, rendering it a promising candidate to be used as a multifunctional carrier. The strategy of combination of multifunctional mesoporous silica materials with bioactive molecules could be a new effective approach to improve their capabilities in the drug delivery.

\section{Introduction}

The design of mesoporous silica nanoparticles (denoted as MSNs) as a multifunctional platform for targeted drug delivery has attracted tremendous interests recently. The unique properties of MSNs-large surface area, large pore volume, tunable pore sizes, and versatile chemistry for surface functionalization-make them serve not only as reservoir for drugs payload but also as motifs for functional groups assembly, thus yielding various MSNs-based multifunctional drug delivery systems (DDSs). For example, to endow MSNsbased DDSs with image tracking and magnetic targeting properties, several composite systems have been employed, such as $\mathrm{Fe}_{3} \mathrm{O}_{4}$-quantum dots (QDs) $[1,2], \mathrm{Fe}_{3} \mathrm{O}_{4}$-organic dye [3], and $\mathrm{Fe}_{3} \mathrm{O}_{4}-\mathrm{NaYF}_{4}: \mathrm{Yb}^{3+}, \mathrm{Er}^{3+} / \mathrm{Tm}^{3+}$ [4]. To improve the dispersibility and inherently low biocompatibility of MSNs-based DDSs under physiological condition, various functional groups (amino, carboxyl, thiol, and phosphonate groups), block copolymers, poly (ethylene glycol) (PEG), and phospholipids have been incorporated [5-13]. To achieve controlled release of drugs in a stimuli-responsive manner, such systems as quantum dots, gold nanoparticles, iron oxide nanoparticles, and dendrimer were utilized as gates to cap the pores of the MSNs and prevent guest drugs from leaking [1417]. To target drug delivery to specific cell populations such as tumoral cells, antibodies or folic acid ligands were grafted on the MSNs $[18,19]$, thus allowing them to selectively bind to the cell surface to trigger receptor-mediated endocytosis. For a satisfactory nanoparticulate delivery device, it would be desirable to successfully combine targeting, imaging, therapeutics, biocompatibility, and other functions into one system. However, even for the magnetic and fluorescent MSNs-based one (denoted as MFMSNs), which are close to the requirements of desired DDSs due to the combination of therapeutics, targeting, and imaging functions, such activities as specific recognition of cell populations are still lacking. Moreover, in many cases, it is not easy to incorporate simultaneously several functional groups into one nanoparticle in a sufficient concentration due to the limitation of the number of attachment sites on the particle surface. Accordingly, how to further enhance the bioactivities of these systems is still a big challenge. Recently, the combination of nanoparticles and biological molecules is of intense interest owing to the synergistic properties offered by such newly synthesized composites [20], thus creating possibility for these multifunctional MSNs to further strengthen their capabilities in drug delivery.

Heparin, polyanionic glycosaminoglycans (GAGs) that naturally cover the surface of all eukaryotic cells, is mainly consisted of alternating units of sulfated glucuronic acid and 
structurally diverse glucosamine derivatives [21]. Heparin is predominantly used as an anticoagulant and is also being investigated as a possible agent to regulate complement activity and inflammation [22-24]. Heparin is also able to inhibit cancer cell angiogenesis, tumor growth, and metastasis [25]. Recently, a variety of research has begun to combine the biological activities of heparin with the useful properties of nanomaterials, thus providing novel and enhanced activities with potential applications ranging from improving anticoagulant activity for anticancer therapy to tissue engineering and biosensors [20]. For example, the presence of heparin on polymeric nanoparticles has been reported to increase their circulation time [26]. The surface modifications of heparin to the magnetite nanoparticles are effective in delaying phagocytosis and preventing blood clotting in vitro [27]. A doxorubicin-(DOX-) loaded heparin-DOCA (deoxycholic acid) conjugate was found to exhibit high suppression of tumor growth compared to free DOX [28]. Though heparin has been conjugated to the surface of the nanoparticles, such as magnetic and metallic nanoparticles, or biodegradable and nondegradable synthetic polymers [20], unfortunately, the conjugate of heparin with MSNs is still lacking. In this study, heparin was covalently bonded to amino-modified MFMSNs (denoted as MFMSN-NH$H_{2}$ ) via amide formation, and then the bioactivity of heparinized MFMSNs (denoted as MFMSN-HP) was assessed via plasma recalcification time (PRT), uptake and release of drugs, binding with growth factors that are capable of targeting recognition of tumor cells, and cytotoxicity assay against tumor cells. The aim of the study is to construct a multifunctional anticancer drug carrier by combining the merits of the two substances.

\section{Experimental Section}

2.1. Materials. Tetraethyl orthosilicate (TEOS), cetyltrimethylammonium bromide (CTAB), 3-aminoprpyltethoxysilane (APTES), heparin sodium (Mw 12,000), doxorubicin, and indomethacin were purchased from Shanghai Chemical Corp. 1-Ethyl-3-(3-dimethylami-nopropyl) carbodiimide hydrochloride (EDC), N-hydroxysuccinimide (NHS), 4-morpholineetha-esulfacid (MES), and fluorescein isothiocyanate (FITC) were obtained from Sigma-Aldrich (St. Louis, MO). Basic fibroblast growth factor (bFGF) was purchased from Pharmacia. All other chemicals are of analytical grade.

2.2. Synthesis of FITC-APTES. A solution of FITC ( $8.6 \mathrm{mg})$ in EtOH $(2 \mathrm{~mL})$ was mixed with APTES $(0.197 \mathrm{~mL})$ and then the mixture was stirred at room temperature for $24 \mathrm{~h}$. The prepared FITC-APTES stock solution was kept at $4^{\circ} \mathrm{C}$.

2.3. Synthesis of MFMSNs-NH . The spherical magnetic $\mathrm{Fe}_{3} \mathrm{O}_{4}$ particles were prepared according to the literature [29]. The core-shell structured MFMSNs were prepared according to the literature $[3,30]$. In a typical procedure, as-prepared $\mathrm{Fe}_{3} \mathrm{O}_{4}$ nanoparticles $(0.1 \mathrm{~g})$ were treated with a mixed solution of ethanol $(20 \mathrm{~mL})$ and $\mathrm{HCl}(0.1 \mathrm{M}, 40 \mu \mathrm{L})$ by ultrasonication for $15 \mathrm{~min}$. Subsequently, the treated nanoparticles were separated by a magnet, washed with ethanol and water several times, and then dispersed in a mixture of CTAB $(0.32 \mathrm{~g})$, deionized water $(50 \mathrm{~mL})$, ammonia solution and ethanol $(70 \mathrm{~mL})$. At last, $0.5 \mathrm{~mL}$ of TEOS, and $50 \mu \mathrm{L}$ of FITCAPTES solution was added to the above reaction solution in sequence. After $10 \mathrm{~min}, 50 \mu \mathrm{L}$ of APTES were added and the solution was stirred for $6 \mathrm{~h}$. The obtained products were collected and separated with a magnet, washed several times with ethanol and water, and dried at $80^{\circ} \mathrm{C}$ for $12 \mathrm{~h}$. The structure-directing agent $\mathrm{CTAB}$ was subsequently removed by ultrasonication in acidic $(\mathrm{HCl})$ ethanol (about $0.12 \mathrm{w} / \mathrm{w}$ ) three times [31]. Thus, the amine-functionalized MFMSNs were obtained.

2.4. Heparinization of MFMSNs. Heparin was immobilized onto MFMSNs using a previously reported protocol. MFMSNs- $\mathrm{NH}_{2}(100 \mathrm{mg})$ were suspended in $0.1 \mathrm{M}$ MES buffer (PH 5.5) and hydrated for $2 \mathrm{~h}$, to which EDC (40.0 mg) and NHS (24.0 mg) were added, and then $1.9 \mathrm{mg}$ of heparin was slowly added. The reaction mixture was incubated by rotating overnight. Samples were collected by centrifugation and then dialyzed against deionized water to get rid of the residual EDC, NHS, and heparin. The obtained products denoted as MFMSNs-HP were lyophilized and kept at $4^{\circ} \mathrm{C}$. The contents of heparin incorporated in the MFMSNs were examined by a toluidine blue assay [32].

2.5. PRT Assays. The PRT assays were performed according to a previously reported method [27]. Fresh blood collected from a healthy human was immediately mixed with $3.8 \mathrm{wt} \%$ sodium citrate solution at a dilution ratio of $9: 1$. It was then centrifuged at $3000 \mathrm{rpm}$ and $8^{\circ} \mathrm{C}$ for $20 \mathrm{~min}$ to obtain the platelet-poor plasma (ppp). $0.1 \mathrm{~mL}$ of ppp was transferred to a clotting tube at $37^{\circ} \mathrm{C}$, and $0.1 \mathrm{~mL}$ saline solution $(0.9 \%$ $\mathrm{NaCl}$ ) was added to the ppp. $0.1 \mathrm{~mL}$ of $0.025 \mathrm{M} \mathrm{CaCl}$ solution was then added, and the stop watch started. The clotting tube was tilted at $15 \mathrm{~s}$ intervals until a firm clot was formed. For investigating the effect of the as-synthesized and heparin-functionalized nanoparticles on the plasma clotting time, $0.1 \mathrm{~mL}$ of different concentrations of MFMSNs- $\mathrm{NH}_{2}$ or MFMSNs-HP solution was added in place of the saline solution. At least three experiments were carried out for each sample and the mean clotting time was reported. The differences between the PRT obtained with saline, MFMSNs$\mathrm{NH}_{2}$, and MFMSNs-HP were analyzed statistically using the two-sample $t$-test. The differences observed between these samples were considered significant at $P<0.05$.

2.6. The Loading/Release of Drugs into/from MFMSNs-HP. MFMSNs-HP (25 mg) was dispersed in DOX PBS solution $\left(1 \mathrm{~mL}, 1 \mathrm{mg} \mathrm{mL}^{-1}\right)$ or IDM ethanol solution $(1 \mathrm{~mL}$, $\left.12 \mathrm{mg} \mathrm{mL}^{-1}\right)$. After stirring for $24 \mathrm{~h}$ under dark conditions, the DOX- or IDM-encapsulated MFMSNs-HP was centrifuged and washed with PBS or ethanol solution. To evaluate the DOX or IDM loading amount and efficiency, the supernatant solutions were collected and the residual DOX or IDM was measured by UV-vis spectroscopy at $480 \mathrm{~nm}$ or $250 \mathrm{~nm}$, respectively. Drug loading efficiencies were expressed as 


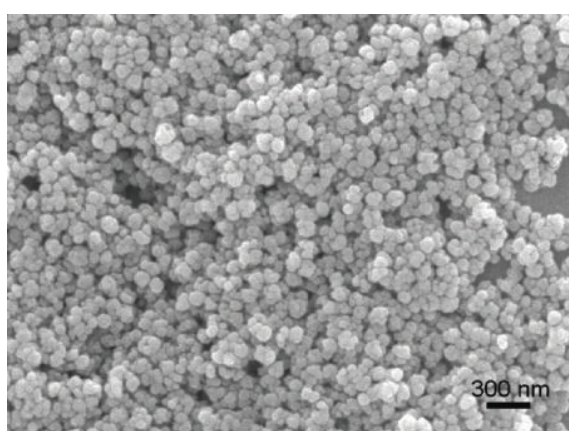

(a)

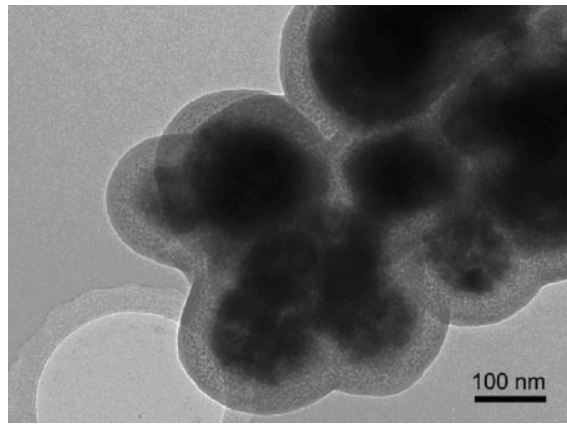

(c)

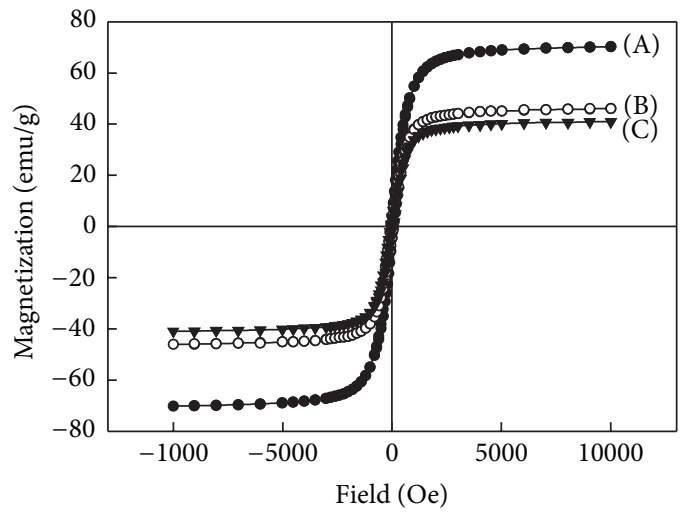

(e)

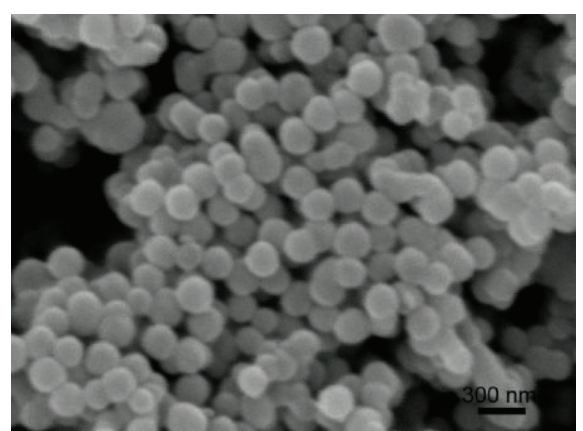

(b)

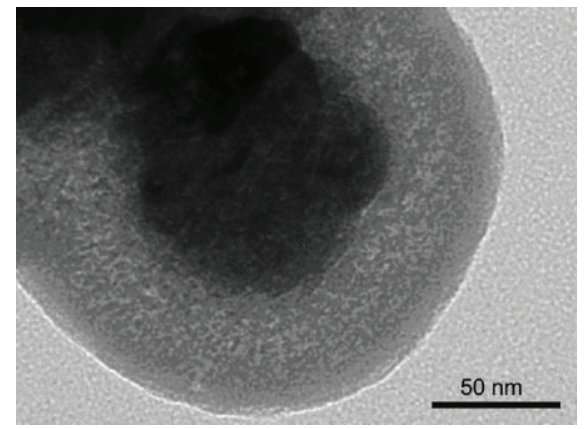

(d)

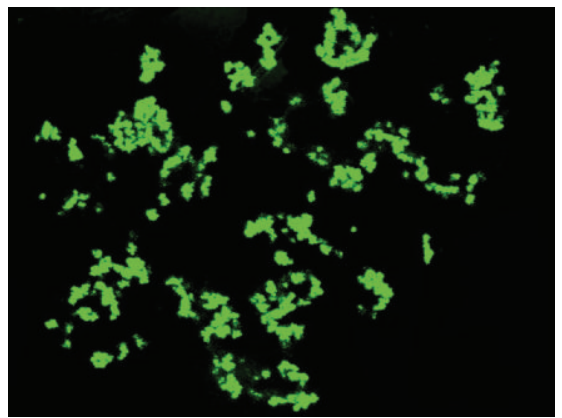

(f)

Figure 1: SEM images of pure $\mathrm{Fe}_{3} \mathrm{O}_{4}$ nanoparticles and MFMSNs ((a), (b)). HRTEM images of MFMSNs ((c)), (d)). The magnetic hysteresis loops (e) of pure $\mathrm{Fe}_{3} \mathrm{O}_{4}$ nanoparticles (A), MFMSNs (B), and MFMSNs-HP (C). Confocal laser scanning microscopy image of MFMSNs (f).

percentages of drugs in MFMSNs-HP nanoparticles with respect to the initial amount of drugs used to prepare the drugs-loaded MFMSNs-HP nanoparticles.

The in vitro release test was performed by immersing the DOX-loaded samples $(25 \mathrm{mg}$ ) in $4 \mathrm{~mL}$ of PBS solution (pH 7.4) and ultrasonification at $40 \mathrm{~W}$ for $1 \mathrm{~min}$. The dispersed DOX-loaded MFMSNs-HP nanoparticles in PBS solution were gently shaken at $37^{\circ} \mathrm{C}$ in a water bath at $180 \mathrm{rpm}$. At given time, the medium was replaced with fresh medium. The amount of the released DOX was determined by UV-vis spectrophotometer.

2.7. The Binding of bFGF to MFMSNs-HP. The binding of bFGF to MFMSNs-HP was monitored by fluorescence titra- tion. Fluorescence spectra were measured with RF-5301PC Shimadzu spectrofluorometer. bFGF and DOX were excited at $278 \mathrm{~nm}$ and $450 \mathrm{~nm}$, respectively. All spectra were recorded at room temperature in $20 \mathrm{mM} \mathrm{pH} 7.4 \mathrm{PBS}$ buffer solutions, MFMSNs-HP titrations were conducted by adding aliquots of either a stock $\left(10 \mathrm{mg} \mathrm{mL}^{-1}\right)$ into $1 \mathrm{~mL}$ of $2 \mu \mathrm{M}$ bFGF solutions or a stock $100 \mu \mathrm{M}$ bFGF solution into $0.5 \mathrm{mg} \mathrm{mL}^{-1}$ MFMSNsHP solutions. After gentle shaking for $5 \mathrm{~min}$, MFMSNs-HP was separated with a magnet, and the supernatant was sent to fluorescence measurement.

2.8. Characterization. Scanning electron microscopy (SEM) images were obtained on a field emission JEOL JSM-6700F 


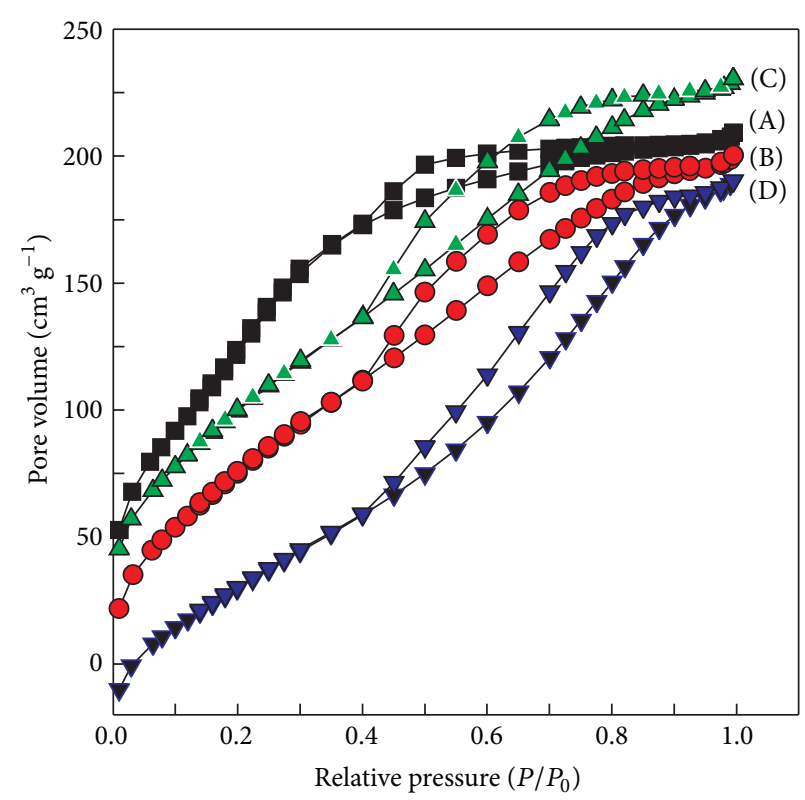

(a)

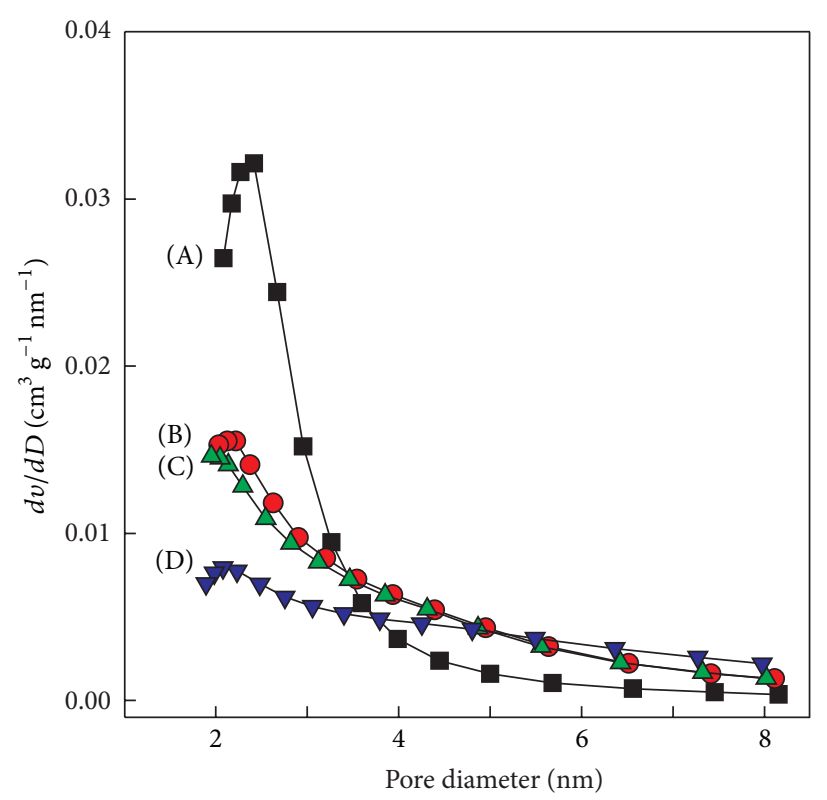

(b)

FIGURE 2: $\mathrm{N}_{2}$ adsorption-desorption isotherms (a) and the corresponding pore size distributions (b) of MFMSNs (A), MFMSNs-HP (B), IDM-loaded MFMSNs-HP (C), and DOX-loaded MFMSNs-HP (D).

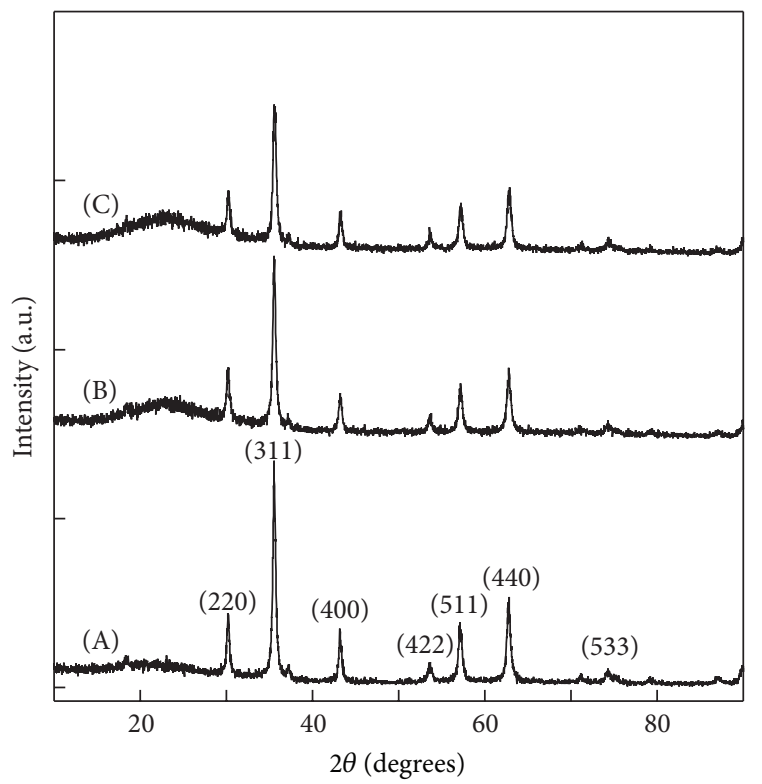

Figure 3: X-ray diffraction patterns of pure $\mathrm{Fe}_{3} \mathrm{O}_{4}$ nanoparticles (A), MFMSNs (B), and MFMSNs-HP (C).

microscope. Transmission electron microscopy (TEM) analyses were conducted on a JEM-2100F electron microscope operating at $200 \mathrm{kV}$. UV-vis spectra were recorded on a UV-3101PC Shimadzu UV-vis spectroscope. For photoluminescence (PL) spectroscopy, an RF-5301PC Shimadzu spectrofluorometer was used. X-ray diffraction (XRD) pat-

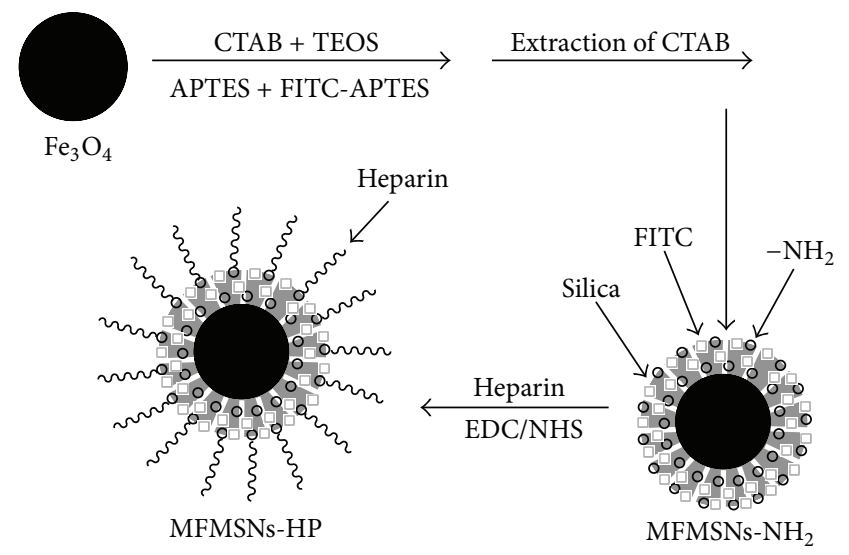

SCHEME 1: The formation process of heparinized mesoporous silica nanoparticles embedded by magnetic and fluorescent groups.

tern was collected using a Rigaku D/Max-2200 PC Xray diffractometer with $\mathrm{Cu}$ target $(40 \mathrm{kV}, 40 \mathrm{~mA})$. Nitrogen adsorption-desorption isotherms at $77 \mathrm{~K}$ were measured on a Micromertitics Tristar 3000 system. X-ray photoelectron spectroscopic (XPS) analysis was performed on an Escalab MKII X-ray photoelectron spectrometer with $\mathrm{Al} \mathrm{K} \alpha$ X-ray source. To compensate for the surface-charging effect, all core-level spectra were referenced to the C 1 s hydrocarbon peak at $284.6 \mathrm{eV}$. Zeta potential of the nanocomposites was measured by Zetasizer (Nano ZS-90, Malvern Instruments). Confocal images were recorded on Olympus FV1000 confocal laser scanning microscope. 
TABLE 1: Structural parameters of MFMSNs, MFMSNs-HP, IDM-loaded MFMSNs-HP, and DOX-loaded MFMSNs-HP.

\begin{tabular}{lccccc}
\hline Sample & $\begin{array}{c}V_{p} \\
\left(\mathrm{~cm}^{2} / \mathrm{g}\right)\end{array}$ & $\begin{array}{c}S_{\mathrm{BET}} \\
\left(\mathrm{m}^{2} / \mathrm{g}\right)\end{array}$ & $\begin{array}{c}D_{p} \\
(\mathrm{~nm})\end{array}$ & $\begin{array}{c}\text { Heparin loading } \\
(\mathrm{wt} \%)\end{array}$ & $\begin{array}{c}\text { Indomethacin loading } \\
(\mathrm{wt} \%)\end{array}$ \\
\hline MFMSNs & 0.33 & 502 & 2.65 & & $\begin{array}{c}\text { DOX loading } \\
(\mathrm{wt} \%)\end{array}$ \\
MFMSNs-HP & 0.35 & 395 & 2.14 & 2 & 0.1787 \\
IDM-loaded MFMSNs-HP & 0.35 & 383 & 2.13 & & 2.5 \\
DOX-loaded MFMSNs-HP & 0.36 & 296 & 2.10 & & \\
\hline
\end{tabular}

${ }^{\mathrm{a}}$ The specific surface area $(S)$ was calculated by the BET equation. ${ }^{\mathrm{b}}$ The pore volume $(V)$ was obtained according to BJH method. ${ }^{\mathrm{c}}$ The pore size $(D)$ was calculated by the $\mathrm{BJH}$ method from $\mathrm{N}_{2}$ desorption isotherms.

TABLE 2: Comparison of PRT obtained in the absence and presence of MFMSNs-HP.

\begin{tabular}{lc}
\hline Sample & PRT [s] \\
\hline Control with saline solution & $205 \pm 20$ \\
Control with 0.1 $\mathrm{mg} \mathrm{mL}^{-1}$ heparin solution & $>1386$ \\
With $0.1 \mathrm{mg} \mathrm{mL}^{-1}$ as-synthesized MFMSNs & $200 \pm 16$ \\
With $0.1 \mathrm{mg} \mathrm{mL}^{-1} \mathrm{MFMSNs}^{-\mathrm{HP}}$ & $631 \pm 29$ \\
With $0.3 \mathrm{mg} \mathrm{mL}^{-1} \mathrm{MMSNS}_{\mathrm{NH}}$-HP & $1386 \pm 25$ \\
\hline
\end{tabular}

\section{Result and Discussion}

The fabrication of MFMSN-HP is presented in Scheme 1. Typically, the as-prepared $\mathrm{Fe}_{3} \mathrm{O}_{4}$ particle was treated by a modified Stöber procedure, to be coated by a silica layer on its surface. Subsequently, cetyltrimethylammonium bromide (CTAB) was selected as the organic template for the formation of the outer mesoporous silica layer on $\mathrm{Fe}_{3} \mathrm{O}_{4}$ particle. To yield more uniform surface coverage of amino and fluorescent groups on mesoporous silica, APTES (3-aminopropyltriethoxysilane) and fluoresceinisothiocyanate-(FITC) derived APTES were embedded into the silica walls by the cocondensation method. After removal of the organic templates, heparin was finally covalently immobilized onto the outer mesoporous silica layer by employing standard carbodiimide chemistry.

SEM images and HRTEM images indicated that pure $\mathrm{Fe}_{3} \mathrm{O}_{4}$ particles prepared by a solvothermal process consist of monodisperse spherical nanoparticles with an average diameter around $100 \mathrm{~nm}$ (Figure 1(a)). The uniform coating of magnetic particles by the inorganic silica with thicknesses of about $50 \mathrm{~nm}$ not only retained the morphological features, but also led to the formation of the core-shell-structured silica spheres (Figures 1(b), 1(c), and 1(d)), which can be clearly distinguished from different electron penetrability between the magnetic cores and silica shells. As shown in Figure 1(d), HRTEM images also indicated that the silica spheres possessed randomly distributed mesopores. The mesoporous characteristics were further demonstrated by $\mathrm{N}_{2}$ adsorption-desorption isotherms, whereas type IV isotherm with a hysteresis loop was exhibited (Figure 2). The pore size calculated using $\mathrm{BJH}$ method was $2.65 \mathrm{~nm}$, and the BET surface area and the total pore volume were $502 \mathrm{~m}^{2} \mathrm{~g}^{-1}$ and $0.33 \mathrm{~cm}^{3} \mathrm{~g}^{-1}$ respectively (Table 1 ). The uniform mesopores along with large surface area are advantageous for drug loading. The X-ray diffraction results indicated that the composites still retained the original crystallinity of magnetic particles (JCPDS number 19-0629), but had a relatively low intensity due to the shielding effect of the silica shell (Figure 3). Field-dependent magnetism at room temperature showed no hysteresis (Figure 1(e)), exhibiting the superparamagnetic features, which is desirable for their applications to targeted drug delivery. In addition, the nanoparticles exhibited strong green fluorescence emission visualized by the confocal microscope images due to the embedding of fluorescent dyes (Figure 1(f)), which allows the drug release system to be easily tracked by fluorescent images. Although these designed nanoparticles have possessed mesoporous, magnetic, and fluorescent features, hopefully the introduction of bioactive heparin into nanoparticles could afford new functions for drug delivery.

XPS was firstly employed to confirm the grafting of heparin onto MSNs. Figures 4(a) and 4(b) show the XPS survey scan spectra of amino-modified MSNs and heparin-modified MSNs. After heparin immobilization, the appearance of $S$ $2 p$ peak indicates the presence of heparin. The $S 2 p$ corelevel spectrum of heparin-modified MSNs is asymmetrical and can be curve-fitted with two peak components at binding energies at 168.8 and $170 \mathrm{eV}$, respectively, owing to the $-\mathrm{OSO}_{3}$ and $-\mathrm{NSO}_{3}$ moieties in heparin (Figure 4(c)) [27]. Herein, it should be mentioned that MSNs without containing FITC was used for the XPS measurement in order to rule out $S$ disturbance of FITC since APTES-or FITC-derived APTES was cocondensed into silica layer of $\mathrm{Fe}_{3} \mathrm{O}_{4} @ \mathrm{nSiO}_{2}$. The $\mathrm{N}$ 1s core-level peaks of heparin-modified MSNs at $398.5 \mathrm{eV}$ and $399.8 \mathrm{eV}$ are attributed to amine nitrogen and amide nitrogen respectively (Figure 4(d)). The peak area of amide nitrogen, is much larger than that of amine nitrogen, suggesting that most of the amine groups have been converted to amide groups after heparin immobilization. Moreover, after heparin loading, the BET surface area of the nanoparticles was decreased to $395 \mathrm{~m}^{2} \mathrm{~g}^{-1}$ (Table 1), and the zeta potential of the nanoparticles at $\mathrm{pH}$ 7.4 PBS buffer solution was reduced from $-16 \mathrm{mV}$ to $-30 \mathrm{mV}$ as well. Therefore, all these results indicated that heparin had been successfully grafted to the nanoparticles, and the loading amount was approximately $2.0 \%(\mathrm{w} / \mathrm{w})$ determined by toluidine blue assay [29].

It is well known that heparin has a variety of bioactivities such as anticoagulant activity. After the immobilization of heparin on the backbone of MFMSNs, its bioactivities 


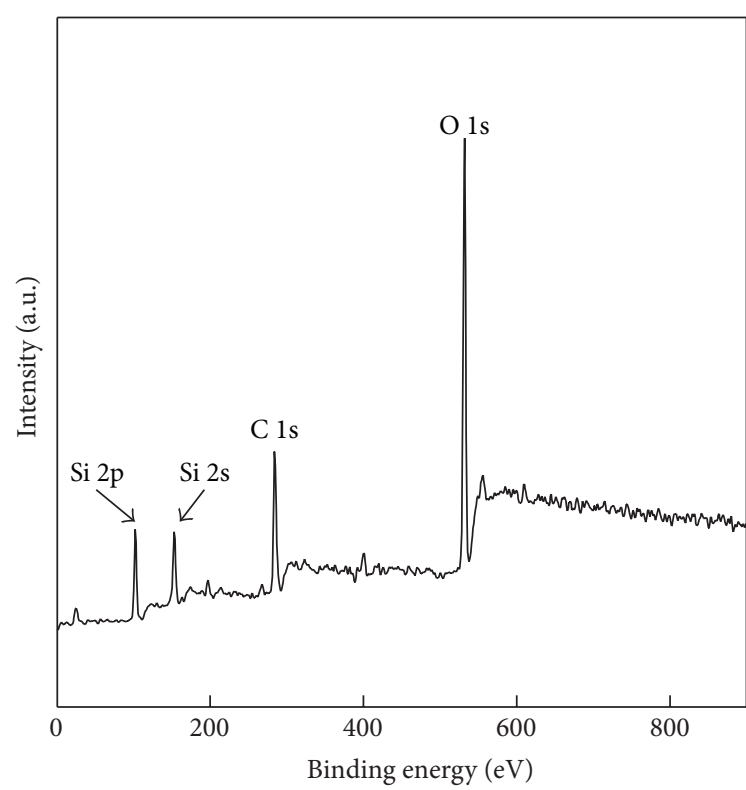

(a)

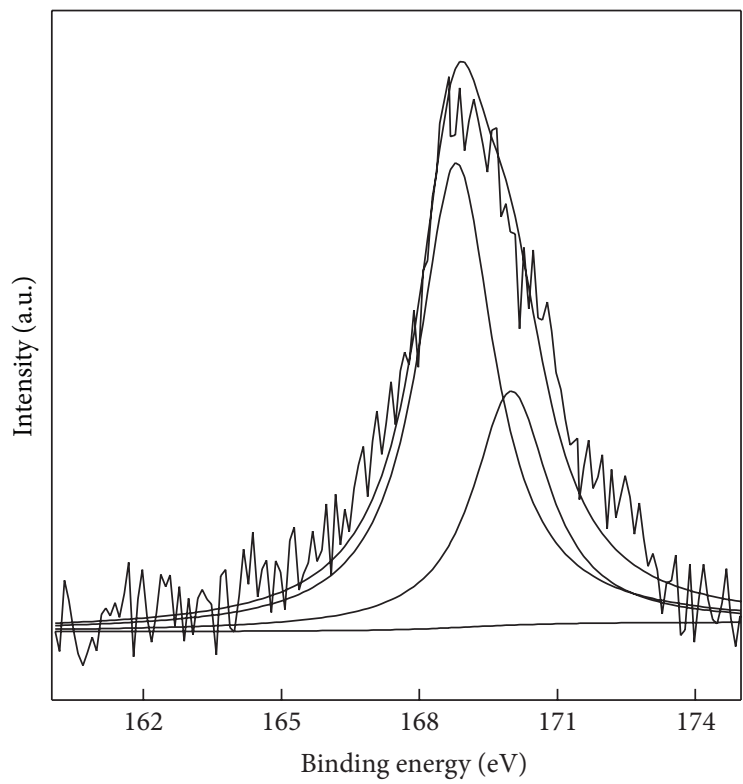

(c)

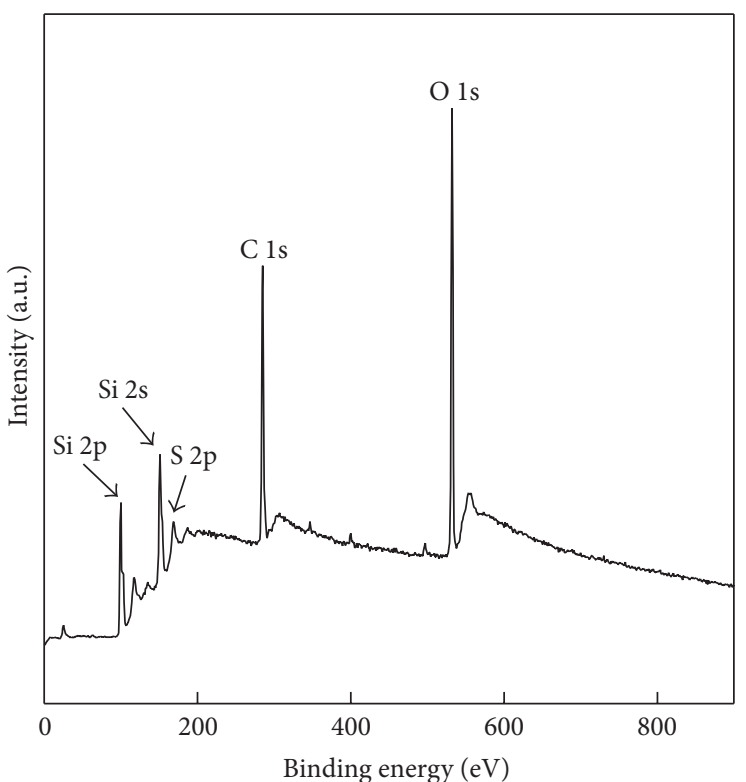

(b)

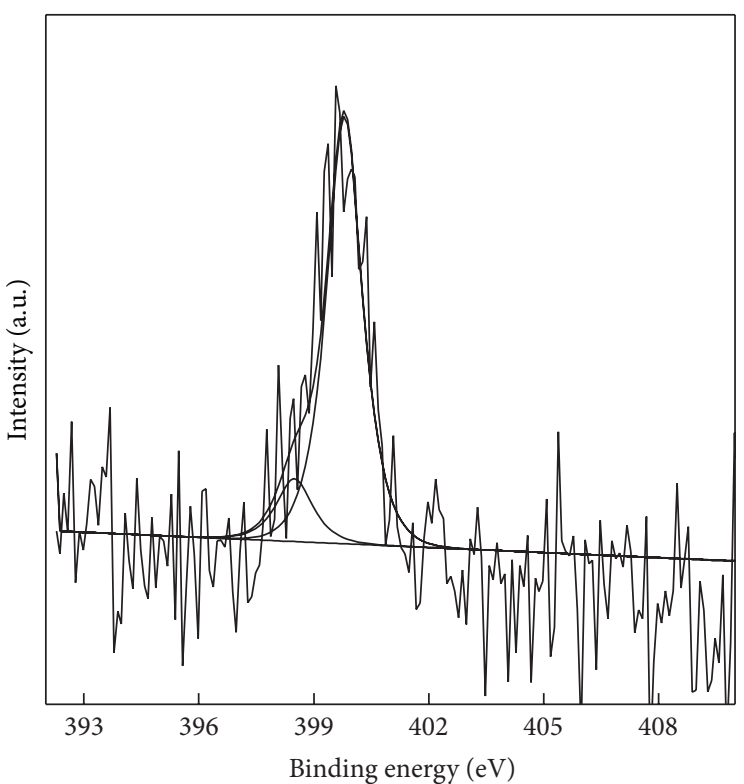

(d)

FIGURE 4: Survey XPS spectra of amino-modified MSNs (a); heparin-modified MSNs (b); XPS S 2p spectrum of heparin-modified MSNs (c); XPS N 1s spectrum of heparin-modified MSNs (d).

are expected to be remained for the following biomedical application. Thus, the assay of plasma recalcification time (PRT) was performed. As heparin is a known anticoagulant, the PRT of MFMSNs-HP dispersion would be expected to be longer than the normal PRT. As can be seen from Table 2, the PRT of $0.1 \mathrm{mg} \mathrm{mL}^{-1}$ of MFMSNs- $\mathrm{NH}_{2}$ did not show a clear difference compared to the blank control $(0.9 \%$ $\mathrm{NaCl}$ ). However, the PRT of $0.1 \mathrm{mg} \mathrm{mL}^{-1}$ of MFMSNs-HP was larger than that of the blank control $(200 \mathrm{~s}$ versus 630 s, $P<0.05$ ), and furthermore, the PRT of MFMSNs$\mathrm{HP}$ can be prolonged to $1380 \mathrm{~s}$ at the concentration of
$0.3 \mathrm{mg} \mathrm{mL}^{-1}$, suggesting that the immobilization of heparin on the MFMSNs increases their anticlotting activity in a dose-dependence style. In a recent report, the anticoagulation activities of multifunctional nanoellipsoids (MFNEs) were evaluated via prothrombin time (PT) and activated partial thromboplastin time (APTT) assays [4]. The result showed that the PT and APTT values of the blood plasma after the exposure to MFNEs did not show a clear difference compared to the blank control (PBS solution), indicating that MFNEs do not cause coagulation. In this regard, the anticoagulation effects of MFMSNs-HP are better than that 


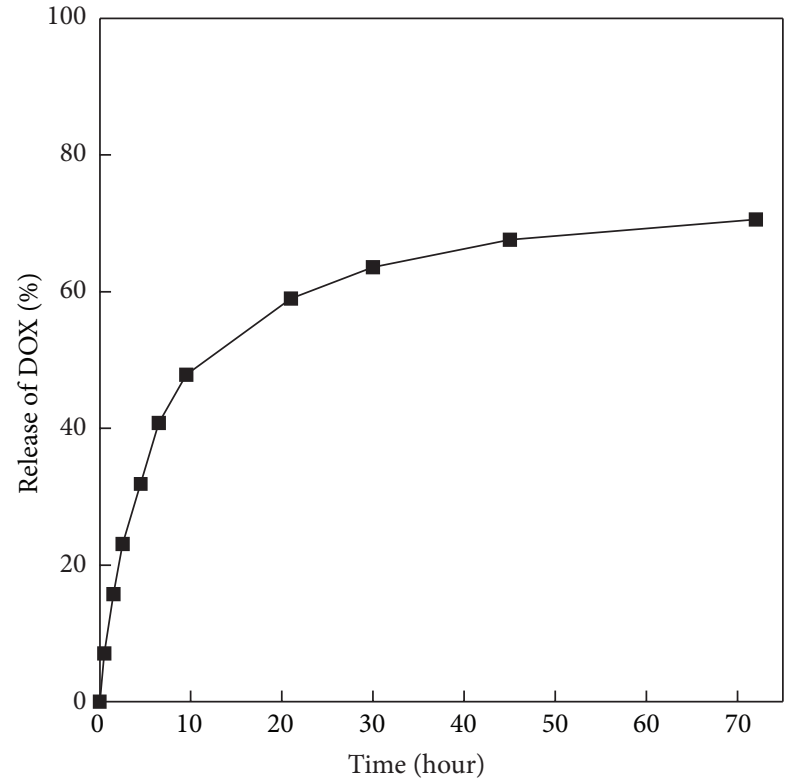

(a)

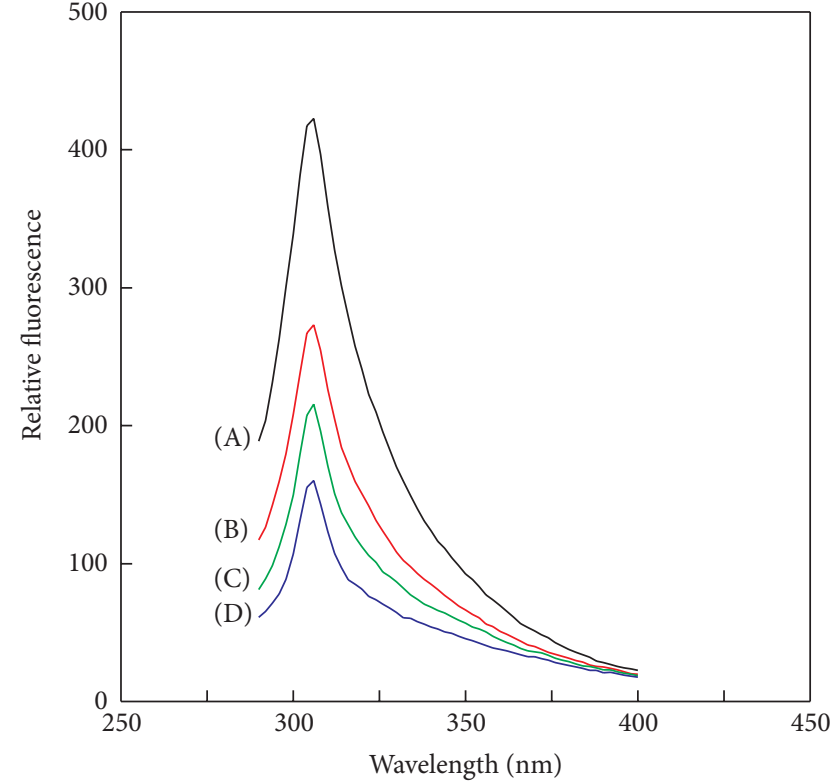

(b)

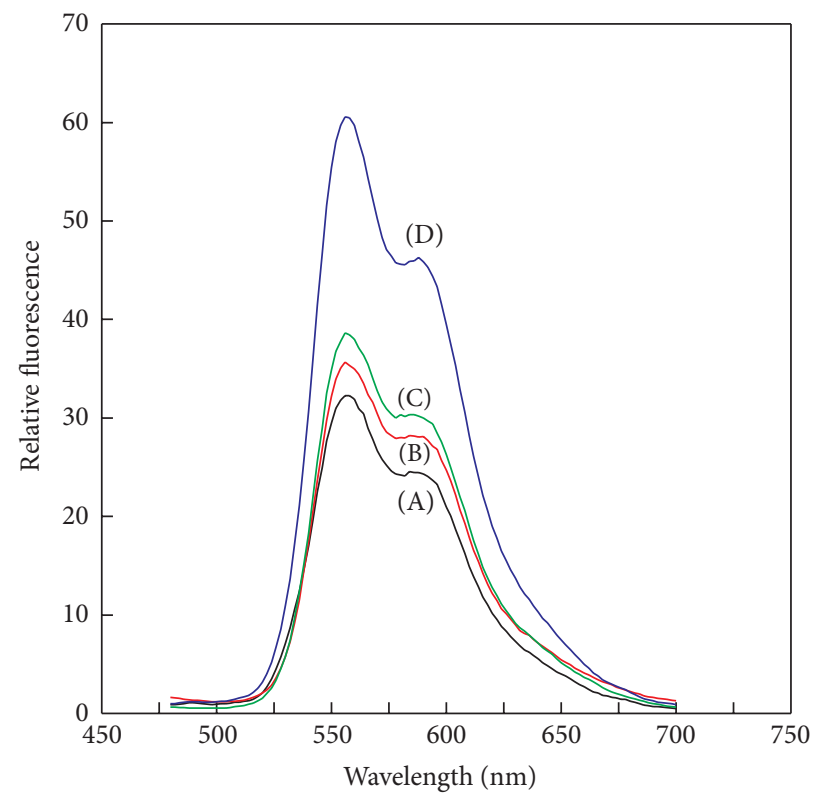

(c)

FIGURE 5: (a) Release profile of DOX from MFMSNs-HP ( $\mathrm{pH}=7.4)$. (b) Fluorescence spectra of bFGF in the absence/presence of MFMSNsHP: free bFGF $(2 \mu \mathrm{M}, 1 \mathrm{~mL})(\mathrm{A}), \mathrm{bFGF}(2 \mu \mathrm{M})+10,20$, and $30 \mu \mathrm{L}$ of MFMSNs-HP $\left(10 \mathrm{mg} \mathrm{mL}^{-1}\right)(\mathrm{B}, \mathrm{C}, \mathrm{D})$. (c) Fluorescence spectra of DOX in the absence/presence of bFGF: free DOX $(1 \mathrm{nmol}, 1 \mathrm{~mL}(\mathrm{~A}), 2 \mathrm{nmol}, 1 \mathrm{~mL}(\mathrm{D}))$, DOX-loaded MFMSNs-HP $\left(0.5 \mathrm{mg} \mathrm{mL} \mathrm{mL}^{-1}, 1 \mathrm{~mL}\right)+10 \mathrm{and}$ $20 \mu \mathrm{L}$ of bFGF $(100 \mu \mathrm{M})(\mathrm{B}, \mathrm{C})$.

of MFNEs but lower than that of heparinized magnetic nanoparticles [27]. The main reason is possibly the lowdensity surface coating of heparin on the mesoporous silica. For heparinized magnetic nanoparticles, the modification of poly(NIPAAM) to the magnetic nanoparticles allowed a large amount of heparin molecules to be immobilized on their surface. Therefore, the strategy of the immobilization of heparin on MFMSNs- $\mathrm{NH}_{2}$ needs to be adjusted to further improve their blood compatibility. The layer-by-layer coating of the MFMSNs- $\mathrm{NH}_{2}$ with negatively charged heparin and positively charged polyelectrolytes might be a better choice.

To explore MFMSNs-HP as a candidate of drug carriers, we selected both doxorubicin (DOX) and indomethacin (IDM) as the model drugs. Positively charged DOX is a typical anticancer drug and negatively charged IDM is a nonsteroidal anti-inflammatory agent. The loaded amount of drugs was quantified with UV-vis spectroscopy by subtracting the supernatant amount from the feeding 
amount. Approximately $0.06 \mathrm{mg}$ of DOX was loaded per milligram of MFMSNs-HP (6.0\% by mass) and the loading of DOX in MFMSNs-HP further decreased the BET surface area of MFMSNs- $\mathrm{NH}_{2}$ to $296 \mathrm{~m}^{2} \mathrm{~g}^{-1}$ (Table 1). While for IDM, the loading value is very low and even could be neglected. $\mathrm{N}_{2}$ adsorption/desorption parameters of IDM-loaded MFMSNsHP do not exhibit notable differences compared to those of free MFMSNs-HP (Table 1), suggesting that nearly no IDM penetrates into the MFMSNs-HP. These results clearly indicated that electrostatic attraction played an important role in the drugs loading. But for the resistance of MFMSNs-HP to hydrophobic IDM, ionic interaction alone is not enough because lots of hydrophobic drugs had been reported to be encapsulated in mesoporous silica for the enhancement of drug dissolution [33]. In the case of MFMSNs-HP, the heparin molecules are probably arranged as a brush-like shell on the surface of nanoparticles, thus allowing their surface more hydrophilic and their zeta potential much lower than that of original materials. The failure of IDM to be attached in the MMSNs-HP could result from both hydrophilic and electrostatic repellences. Therefore, MFMSNs-HP could selectively bind with those of positively charged and hydrophilic drugs. Subsequently, the release of DOX from MFMSNs-HP was performed at $\mathrm{pH} 7.4$ of PBS solution. As shown in Figure 5(a), after the burst release of DOX within the initial $8 \mathrm{~h}$, the release process could be sustained to more than $72 \mathrm{~h}$. This is rather promising for the application of MFMSNs-HP as drug carriers.

Ligand-directed delivery of drugs to tumors by binding to cancer cell-surface receptors has found success in current DDSs [34]. Growth factors such as basic fibroblast factor (bFGF) or epidermal growth factor (EGF) as one of the represented targeting ligands for tumor cells have been incorporated into several drug carriers $[35,36]$. The mechanism of growth-factors-derived DDSs is mainly involved in specific affinity of these growth factors with their cognate cell-surface receptors that are found to be overexpressed in numerous tumor cells in comparison to normal cells. Heparin has high affinity with bFGF and the binding of heparin stabilizes the proteins against proteolysis and maintains their conformation. Such advantage along with the brushlike arrangement of heparin at the exterior of MFMSNs-HP gives us a hint to further modify DOX-loaded MFMSNs-HP with bFGF. The binding of bFGF to DOXloaded MFMSNs-HP was verified by fluorescence titration, where the endogenous fluorescence of protein was quenched little by little with an increase in the amount of DOX-loaded materials (Figure 5(b)). Herein, it is necessary to investigate whether the binding reaction brought about the leakage of drugs from materials. To study this problem, $0.5 \mathrm{mg}$ equal of materials containing $50 \mathrm{nmol}$ of DOX was mixed with varied concentration bFGF in $1 \mathrm{~mL}$ of PBS solution. After incubation at the same time intervals ( $5 \mathrm{~min}$ ) as those of the binding reaction, the fluorescence of DOX was measured. As shown in Figure 5(c), the fluorescence intensity of all these samples was in the middle of that of $1 \mu \mathrm{M}$ and $2 \mu \mathrm{M}$ of free DOX, indicating that the immobilization of bFGF on the MFMSNsHP nearly has no influence on the loaded drugs. Thus, it is feasible for MFMSNs-HP to load anticancer drugs and growth factors simultaneously, suggesting the potential of the obtained nanoparticles in tumor targeted drug delivery.

\section{Conclusion}

A number of drug delivery systems based on functionalized mesoporous silica nanoparticles had been developed recently [37-41]. In the present work, a novel system based on heparinized multifunctional mesoporous silica nanoparticles was also described. The system not only maintains intrinsic functions of bare magnetic and fluorescent mesoporous silica materials such as targeting, imaging, and sustained release of drugs, but also generates several novel activities such as the enhancement of biocompatibility, selective loading drugs, and dual loading of anticancer drug and bFGF. The strategy of combination of multifunctional mesoporous silica materials with bioactive molecules could be a new effective approach to improve their capabilities in the drug delivery. Besides drug delivery, the properties of MFMSNs-HPs may allow it to be useful in other biomedical applications, such as magnetic resonance (MR) and fluorescence imaging, and affinity purification of heparin-binding growth factors. Additionally, there is a problem that should be mentioned. The incorporation of anticoagulant heparin into mesoporous silica nanoparticles not only endows this drug delivery system with biocompatibility but may also introduce the risk of inducing hemorrhage at the time of intravenous administration. Therefore, further biochemical evaluation for this system by in vivo/vitro assays is needed, but the aggregates of the obtained nanoparticles will make some investigations not to be carried out, such as cells uptake and cells toxicity. The optimization of reaction conditions to improve the structure of this material is underway.

\section{References}

[1] J. Kim, J. E. Lee, J. Lee et al., "Magnetic fluorescent delivery vehicle using uniform mesoporous silica spheres embedded with monodisperse magnetic and semiconductor nanocrystals," Journal of the American Chemical Society, vol. 128, no. 3, pp. 688-689, 2006.

[2] Y. Chen, H. Chen, S. Zhang et al., "Multifunctional mesoporous nanoellipsoids for biological bimodal imaging and magnetically targeted delivery of anticancer drugs," Advanced Functional Materials, vol. 21, no. 2, pp. 270-278, 2011.

[3] J. Kim, H. S. Kim, N. Lee et al., "Multifunctional uniform nanoparticles composed of a magnetite nanocrystal core and a mesoporous silica shell for magnetic resonance and fluorescence imaging and for drug delivery," Angewandte Chemie International Edition, vol. 47, no. 44, pp. 8438-8441, 2008.

[4] S. Gai, P. Yang, C. Li et al., "Synthesis of magnetic, upconversion luminescent, and mesoporous core-shell-structured nanocomposites as drug carriers," Advanced Functional Materials, vol. 20, no. 7, pp. 1166-1172, 2010.

[5] J. Kim, J. E. Lee, J. Lee et al., "Generalized fabrication of multifunctional nanoparticle assemblies on silica spheres," Angewandte Chemie International Edition, vol. 45, no. 29, pp. 4789-4793, 2006.

[6] R. Kumar, I. Roy, T. Y. Ohulchanskyy et al., "Covalently dye-linked, surface-controlled, and bioconjugated organically 
modified silica nanoparticles as targeted probes for optical imaging," ACS Nano, vol. 2, no. 3, pp. 449-456, 2008.

[7] S. Sadasivan, D. Khushalani, and S. Mann, "Synthesis and shape modification of organo-functionalised silica nanoparticles with ordered mesostructured interiors," Journal of Materials Chemistry, vol. 13, no. 5, pp. 1023-1029, 2003.

[8] R. P. Bagwe, L. R. Hilliard, and W. Tan, "Surface modification of silica nanoparticles to reduce aggregation and nonspecific binding," Langmuir, vol. 22, no. 9, pp. 4357-4362, 2006.

[9] Q. Huo, J. Liu, L. Q. Wang, Y. Jiang, T. N. Lambert, and E. Fang, "A new class of silica cross-linked micellar core-shell nanoparticles," Journal of the American Chemical Society, vol. 128, no. 19, pp. 6447-6453, 2006.

[10] S. Jo and K. Park, "Surface modification using silanated poly(ethylene glycol)s," Biomaterials, vol. 21, no. 6, pp. 605-616, 2000.

[11] C. Y. Yang, S. J. Cai, H. Liu, and C. Pidgeon, "Immobilized artificial membranes-screens for drug membrane interactions," Advanced Drug Delivery Reviews, vol. 23, no. 1-3, pp. 229-256, 1997.

[12] J. Liu, A. Stace-Naughton, X. Jiang, and C. J. Brinker, "Porous nanoparticle supported lipid bilayers (protocells) as delivery vehicles," Journal of the American Chemical Society, vol. 131, no. 4, pp. 1354-1355, 2009.

[13] L. S. Wang, L. C. Wu, S. Y. Lu et al., "Biofunctionalized phospholipid-capped mesoporous silica nanoshuttles for targeted drug delivery: improved water suspensibility and decreased nonspecific protein binding," ACS Nano, vol. 4, no. 8, pp. 4371-4379, 2010.

[14] C. Y. Lai, B. G. Trewyn, D. M. Jeftinija et al., "A mesoporous silica nanosphere-based carrier system with chemically removable CdS nanoparticle caps for stimuli-responsive controlled release of neurotransmitters and drug molecules," Journal of the American Chemical Society, vol. 125, no. 15, pp. 4451-4459, 2003.

[15] F. Torney, B. G. Trewyn, V. S. Y. Lin, and K. Wang, "Mesoporous silica nanoparticles deliver DNA and chemicals into plants," Nature Nanotechnology, vol. 2, no. 5, pp. 295-300, 2007.

[16] S. Giri, B. G. Trewyn, M. P. Stellmaker, and V. S. Y. Lin, "Stimuliresponsive controlled-release delivery system based on mesoporous silica nanorods capped with magnetic nanoparticles," Angewandte Chemie International Edition, vol. 44, no. 32, pp. 5038-5044, 2005.

[17] D. R. Radu, C. Y. Lai, K. Jeftinija, E. W. Rowe, S. Jeftinija, and V. S. Y. Lin, "A polyamidoamine dendrimer-capped mesoporous silica nanosphere-based gene transfection reagent," Journal of the American Chemical Society, vol. 126, no. 41, pp. 13216-13217, 2004.

[18] C. P. Tsai, C. Y. Chen, Y. Hung, F. H. Chang, and C. Y. Mou, "Monoclonal antibody-functionalized mesoporous silica nanoparticles (MSN) for selective targeting breast cancer cells," Journal of Materials Chemistry, vol. 19, no. 32, pp. 5737-5743, 2009.

[19] J. M. Rosenholm, A. Meinander, E. Peuhu et al., "Targeting of porous hybrid silica nanoparticles to cancer cells," ACS Nano, vol. 3, no. 1, pp. 197-206, 2009.

[20] M. M. Kemp and R. J. Linhardt, "Heparin-based nanoparticles," Wiley Interdisciplinary Reviews: Nanomedicine and Nanobiotechnology, vol. 2, no. 1, pp. 77-87, 2010.

[21] M. Ishihara and K. Ono, "Structure and function of heparin and heparan sulfate; Heparinoid library and modification of FGFactivities," Trends in Glycoscience and Glycotechnology, vol. 10, no. 52, pp. 223-233, 1998.
[22] H. P. T. Ekre, B. Fjellner, and O. Hagermark, "Inhibition of complement dependent experimental inflammation in human skin by different heparin fractions," International Journal of Immunopharmacology, vol. 8, no. 3, pp. 277-286, 1986.

[23] M. D. Sharath, Z. M. Merchant, and Y. S. Kim, "Small heparin fragments regulate the amplification pathway of complement," Immunopharmacology, vol. 9, no. 2, pp. 73-80, 1985.

[24] J. M. Weiler, R. E. Edens, R. J. Linhardt, and D. P. Kapelanski, "Heparin and modified heparin inhibit complement activation in vivo," Journal of Immunology, vol. 148, no. 10, pp. 3210-3215, 1992.

[25] S. M. Smorenburg and C. J. F. Van Noorden, "The complex effects of heparins on cancer progression and metastasis in experimental studies," Pharmacological Reviews, vol. 53, no. 1, pp. 93-105, 2001.

[26] C. J. van Oss, "Phagocytosis as a surface phenomenon," Annual Review of Microbiology, vol. 32, pp. 19-39, 1978.

[27] S. C. Wuang, K. G. Neoh, E. T. Kang, D. W. Pack, and D. E. Leckband, "Heparinized magnetic nanoparticles: in-vitro assessment for biomedical applications," Advanced Functional Materials, vol. 16, no. 13, pp. 1723-1730, 2006.

[28] K. Park, G. Y. Lee, Y. S. Kim et al., "Heparin-deoxycholic acid chemical conjugate as an anticancer drug carrier and its antitumor activity," Journal of Controlled Release, vol. 114, no. 3, pp. 300-306, 2006.

[29] H. Deng, X. Li, Q. Peng, X. Wang, J. Chen, and Y. Li, "Monodisperse magnetic single-crystal ferrite microspheres," Angewandte Chemie International Edition, vol. 44, no. 18, pp. 2782-2785, 2005.

[30] X. Guo, Y. Deng, D. Gu, R. Che, and D. Zhao, "Synthesis and microwave absorption of uniform hematite nanoparticles and their core-shell mesoporous silica nanocomposites," Journal of Materials Chemistry, vol. 19, no. 37, pp. 6706-6712, 2009.

[31] K. Möller, J. Kobler, and T. Bein, "Colloidal suspensions of nanometer-sized mesoporous silica," Advanced Functional Materials, vol. 17, no. 4, pp. 605-612, 2007.

[32] H. J. Chung, H. K. Kim, J. J. Yoon, and T. G. Park, "Heparin immobilized porous PLGA microspheres for angiogenic growth factor delivery," Pharmaceutical Research, vol. 23, no. 8, pp. 1835-1841, 2006.

[33] M. Van Speybroeck, V. Barillaro, T. D. Thi et al., "Ordered mesoporous silica material SBA-15: a broad-spectrum formulation platform for poorly soluble drugs," Journal of Pharmaceutical Sciences, vol. 98, no. 8, pp. 2648-2658, 2009.

[34] R. Sinha, G. J. Kim, S. Nie, and D. M. Shin, "Nanotechnology in cancer therapeutics: bioconjugated nanoparticles for drug delivery," Molecular Cancer Therapeutics, vol. 5, no. 8, pp. 19091917, 2006.

[35] A. A. Bhirde, V. Patel, J. Gavard et al., "Targeted killing of cancer cells in vivo and in vitro with EGF-directed carbon nanotubebased drug delivery," ACS Nano, vol. 3, no. 2, pp. 307-316, 2009.

[36] L. Cai, N. Qiu, X. Li et al., "A novel truncated basic fibroblast growth factor fragment-conjugated poly (ethylene glycol)cholesterol amphiphilic polymeric drug delivery system for targeting to the FGFR-overexpressing tumor cells," International Journal of Pharmaceutics, vol. 408, no. 1-2, pp. 173-182, 2011.

[37] Y. Furukawa, T. Ishiwata, K. Sugikawa, K. Kokado, and K. Sada, "Nano- and microsized cubic gel particles from cyclodextrin metal-organic frameworks," Angewandte Chemie International Edition, vol. 51, no. 42, pp. 10566-10569, 2012. 
[38] M. Vallet-Regí, F. Balas, and D. Arcos, "Mesoporous materials for drug delivery," Angewandte Chemie International Edition, vol. 46, no. 40, pp. 7548-7558, 2007.

[39] E. Bringas, Ö. Köysüren, D. V. Quach et al., “Triggered release in lipid bilayer-capped mesoporous silica nanoparticles containing SPION using an alternating magnetic field," Chemical Communications, vol. 48, no. 45, pp. 5647-5649, 2012.

[40] E. Climent, R. Martínez-Máñez, F. Sancenón et al., "Controlled delivery using oligonucleotide-capped mesoporous silica nanoparticles," Angewandte Chemie International Edition, vol. 49, no. 40, pp. 7281-7283, 2010.

[41] E. Aznar, M. D. Marcos, R. Martínez-Máñez et al., "pH- and photo-switched release of guest molecules from mesoporous silica supports," Journal of the American Chemical Society, vol. 131, no. 19, pp. 6833-6843, 2009. 

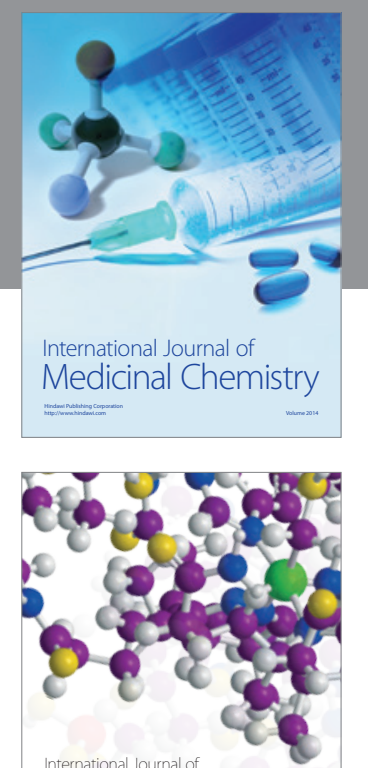

\section{Carbohydrate} Chemistry

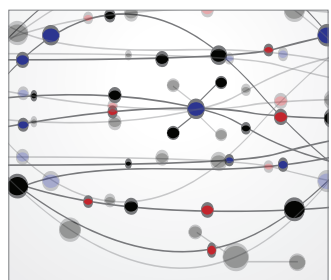

The Scientific World Journal
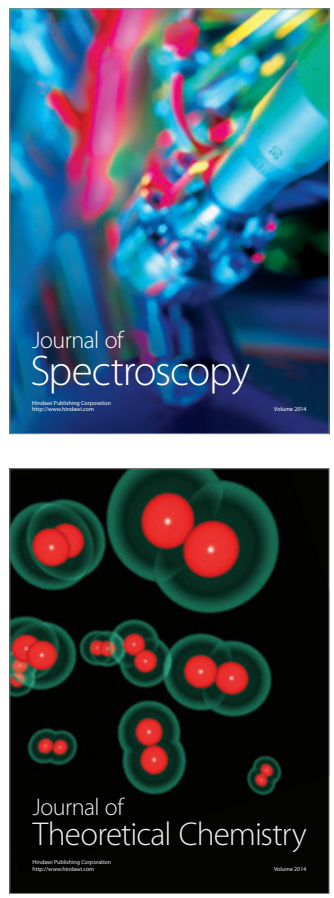
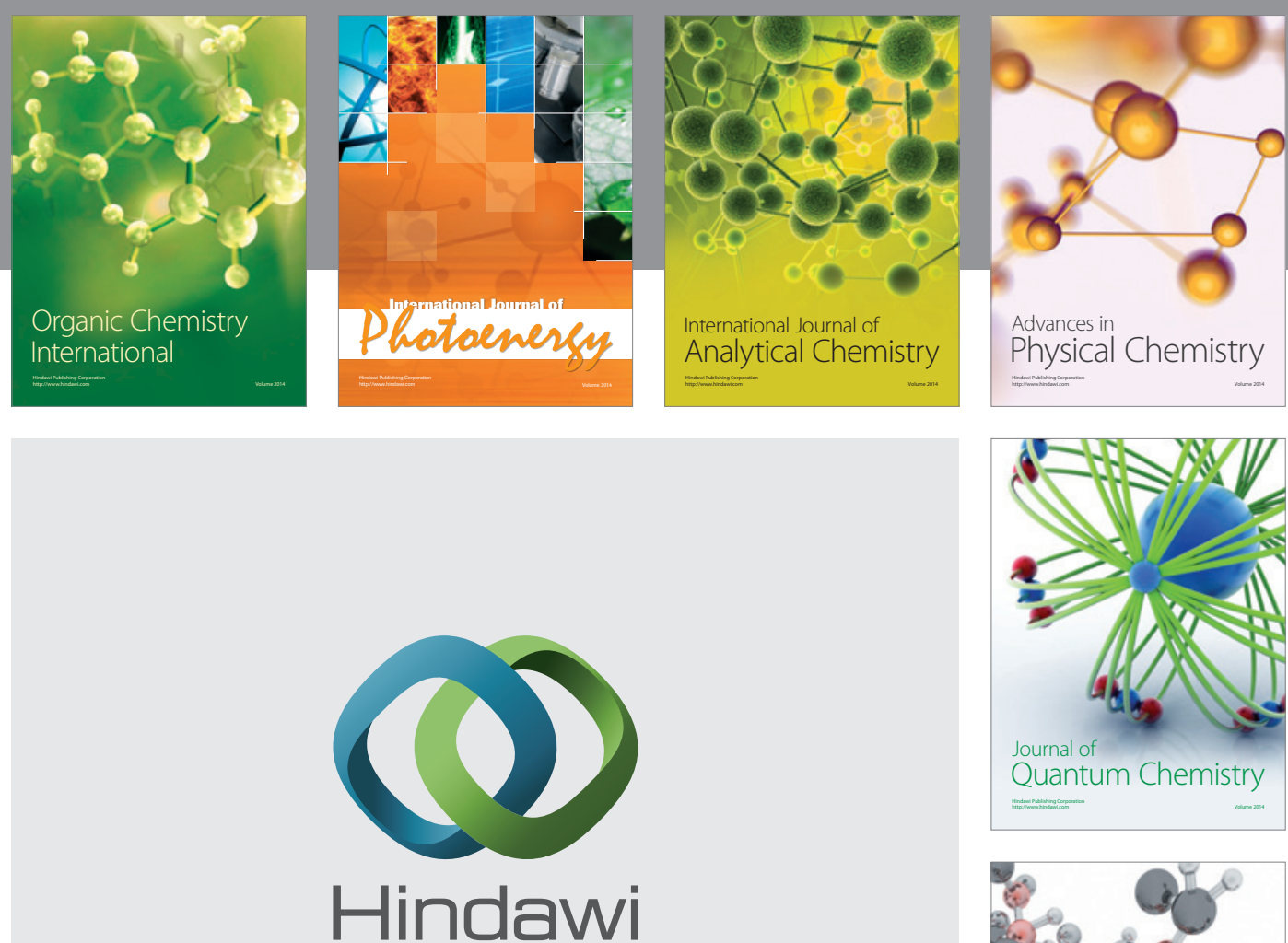

Submit your manuscripts at

http://www.hindawi.com

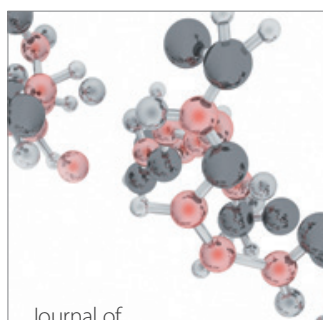

Analytical Methods

in Chemistry

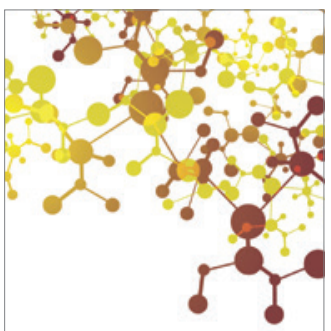

Journal of

Applied Chemistry

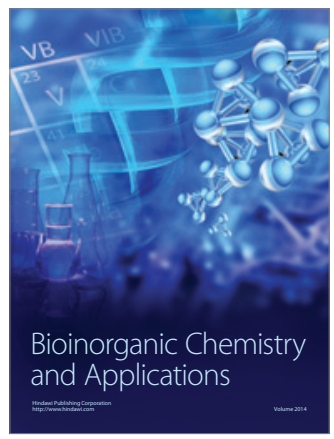

Inorganic Chemistry
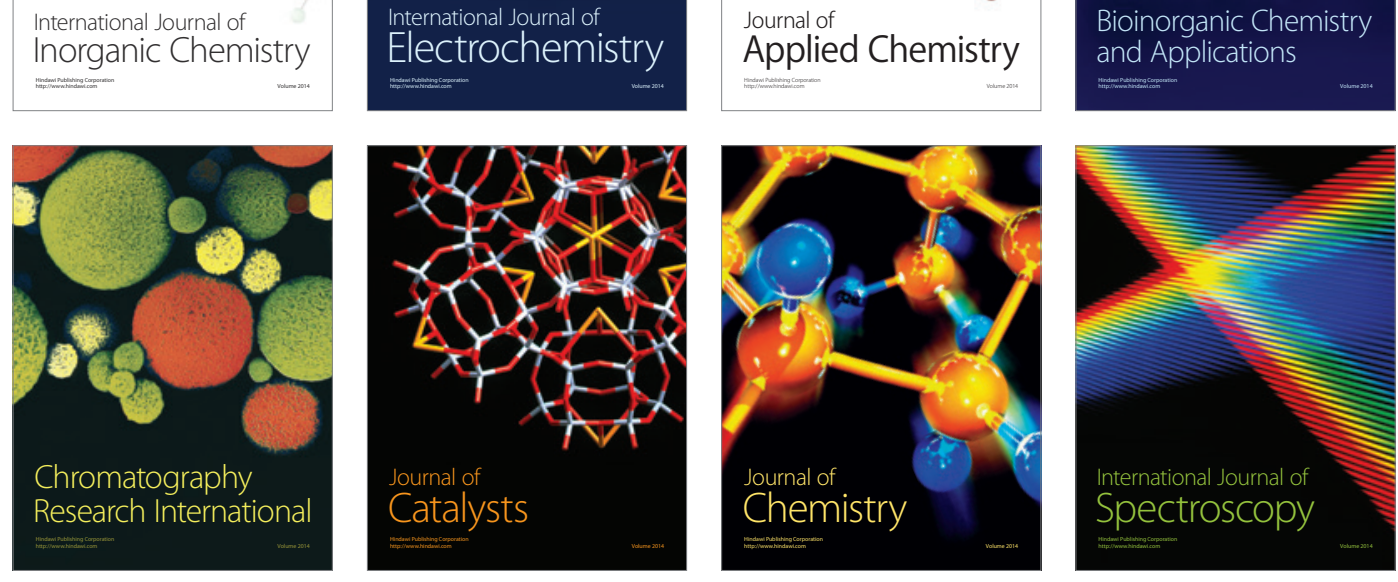